\title{
The impact of order with radiation therapy in stage IIIA pathologic N2 NSCLC patients: a population-based study
}

\author{
Hongxia Duan, Long Liang, Shuanshuan Xie ${ }^{*}$ and Changhui Wang*
}

\begin{abstract}
Background: The aim of this study was to investigate the optimal order of radiation therapy in patients affected by stage IIIA pathologic N2 (IIIA/N2) non-small-cell lung cancer (NSCLC) and to identify its potential risk factors.

Methods: 17,654 (8786 men and 8868 women) diagnosed with NSCLC stage IIIA-N2 from 2004 to 2015 patients were identified in the Surveillance, Epidemiology, and End Results (SEER) database. Among the relevant clinical parameters, we evaluated overall survival (OS), lung cancer-specific survival (LCSS) and other variables such as age, sex and tumor size in patients who were treated with different combinations of surgery and radiotherapy strategies.

Results: We discovered that surgery benefit in younger IIIA/N2 NSCLC patients (age $\leq 75$ ), and compared with surgery only, preoperative radiotherapy significantly improved the survival rate most $(p<0.001)$. When we performed the OS and LCSS analysis in the subgroup of patients' age $>75$ years old, who underwent postoperative radiotherapy (PORT) had the highest survival rate $(p<0.001)$. Multivariate analyses showed that the following parameters had a negative impact on survival: female sex, older age, no chemotherapy, large tumor size, high tumor grade, no surgery or radiotherapy.

Conclusions: In IIIA/N2 NSCLC patients, surgery, radiotherapy and chemotherapy were associated with improved OS and LCSS. Younger patients underwent surgical resection and chemotherapy, the main population we studied, benefited most from preoperative radiotherapy in all orders with radiation therapy $(p<0.001)$. In patients more than 75 years old, there was no clear benefit from only surgery, and PORT was recommended in case of having surgery.
\end{abstract}

Keywords: Non-small-cell lung carcinoma, Survival, Radiotherapy, Surgery, SEER

\section{Background}

Lung cancer is the leading cause of cancer-related mortality worldwide [1]. Lung cancer includes small cell lung cancer (SCLC) and non-small cell lung cancer (NSCLC), the major type of NSCLC are adenocarcinoma (AD) and squamous cell carcinoma (SQCC). In patients diagnosed with lung cancer, $15 \%$ are stage IIIA NSCLC [2-4], while stage IIIA pathologic N2 (IIIA/N2) account for

\footnotetext{
* Correspondence: xieshuanshuan@aliyun.com; 18717774084@163.com
Department of Respiratory Medicine, Shanghai Tenth People's Hospital,

* Correspondence: xieshuanshuan@aliyun.com; 18717774084@163.com
Department of Respiratory Medicine, Shanghai Tenth People's Hospital, Tongji University School of Medicine, \#301, Mid Yanchang Rd, Shanghai 200072, China
}

$50 \%$ of the locally advanced NSCLCs cases [5-7]. NSCL $\mathrm{C}$ patients in IIIA stage having a tumor size T1-T2 (T2: tumor $>3 \mathrm{~cm}$ and $\leq 5 \mathrm{~cm}$ ) and M0 (without distant metastasis), along with ipsilateral mediastinal and/or subcarinal lymph node (N2), are diagnosed as IIIA/N2 NSCLC according to the 8th edition TNM Stage Classification [8]. $\mathrm{N} 2$ are classified into three different groups: occult N2, resectable N2, and non-resectable N2 [9]. Therefore, the optimal treatment for IIIA/N2 NSCLC is still controversial because stage IIIA/N2 NSCLC patients form a very broad and diverse population $[10,11]$.

C C The Author(s). 2020 Open Access This article is licensed under a Creative Commons Attribution 4.0 International License, which permits use, sharing, adaptation, distribution and reproduction in any medium or format, as long as you give appropriate credit to the original author(s) and the source, provide a link to the Creative Commons licence, and indicate if changes were made. The images or other third party material in this article are included in the article's Creative Commons licence, unless indicated otherwise in a credit line to the material. If material is not included in the article's Creative Commons licence and your intended use is not permitted by statutory regulation or exceeds the permitted use, you will need to obtain permission directly from the copyright holder. To view a copy of this licence, visit http://creativecommons.org/licenses/by/4.0/ The Creative Commons Public Domain Dedication waiver (http://creativecommons.org/publicdomain/zero/1.0/) applies to the data made available in this article, unless otherwise stated in a credit line to the data. 
Currently, surgery is still the standard treatment of early-stage NSCLC, but the 5 year survival rate is only 50 to $60 \%$ [6], with a risk of locoregional recurrence of $20-40 \%$ in node-positive patients [12]. Thus, radiotherapy and/or chemotherapy combined with surgery represent the current therapeutic options for these patients. Adjuvant chemotherapy was considered to enhance survival in IIIA NSCLC patients with surgery [13, 14], but $20-40 \%$ of patients still had a local tumor failure [12]. Thus, radiotherapy, including preoperative radiotherapy and postoperative radiotherapy (PORT) is important and necessary.

Since tumor size, lymph node involvement, and comorbidities can widely vary among patients, the idea of having a universal treatment plan for stage IIIA/N2 NSCLC patients seems not feasible. While some studies confirmed that preoperative radiotherapy significantly improve survival $[15,16]$, other studies showed PORT demonstrated better survival instead [17, 18]. Thus, by retrospectively studying the outcomes of IIIA/N2 NSCL $\mathrm{C}$ patients that underwent surgery with either pre- or post-operative radiotherapy or both, we sought to answer the question of which strategy is ideal. Since prospective clinical study are lacking, we perform a retrospective study by using data from the Surveillance, Epidemiology, and End Results (SEER) database to determine which clinical parameters have an impact in the therapy outcome and to provide clinicians and patients more information to make an informed decision.

\section{Methods}

\section{Data source}

We used the US National Cancer Institute's SEER database, which contain data from 18 registered cancer institute covering nearly $26 \%$ of the total US population [19]. The database coverage which is considered an accurate statistical representation of the U.S. population affected with cancer [20]. SEER*-Stat software, version 8.3.2, was used to extract data from the database.

\section{Cohort}

The cohort include patients who were pathologically diagnosed with lung adenocarcinoma (AD) (histological codes 8244, 8245, 8250-8255, 8260, 8290, 8310, 8323, 8333, 8480, 8481, 8490, 8507, 8550, 8570, 8571, 8574, and 8576), squamous cell carcinoma (SQCC) (histologic codes 8052, 8070-8075, 8083, 8084, 8123), and large cell carcinoma (LCC) (histological codes 8012-8014, 8046, $8050,8003,8004,8022,8031-8035,8082,8200,8240$, $8249,8430,8560,8562,8980)$ during a 10 -year period, from 2004 to 2015. Patients graded as stage T1-2 and $\mathrm{N} 2$ were included in this study, while those having a previous malignant disease or distant metastasis were excluded, along with patients who died within 30 days after surgery. Another exclusion criteria was the lack of complete information for the following parameters: age, complete staging, tumor size and location, regional LN examination results, histology, differentiation grade, cause of death, and survival period. Figure 1 showed the detailed case selection process.

\section{Covariates}

Demographic parameters included age, sex, race, insurance coverage, marital status, years of diagnosis, region, education, and median household income. Tumor characteristics included size, histology, $\mathrm{T}$ stage (based on the Eighth Edition Lung Cancer Stage Classification), primary site, and pathologic differentiation grade and laterality.

\section{Statistical analysis}

Pearson's chi-square test was used to assess the baseline parameters and to evaluate the association between the groups. The Kaplan-Meier method was used to generate survival curves, the log-rank test was used to examine the differences in survival among subgroups and multivariate Cox Proportional Hazards Analysis was used to examine the effects of multiple potential prognostic factors on survival. Overall survival (OS) and lung cancerspecific survival (LCSS) were the endpoint measurements. OS was calculated from diagnosis to death from any cause, while LCSS was calculated from the time of diagnosis to death from lung cancer. OS and LCSS were estimated using follow-up data through 2017 and compared in different groups by using the Kaplan-Meier method. All tests were two-sided and $p<0.05$ was considered to be significant. All analyses were performed using the SPSS software, version 22.0 (SPSS Inc. Chicago, IL).

\section{Results}

\section{Baseline cohort characteristics}

Based on the inclusion criteria, this study cohort was formed by 17,654 IIIA/N2 NSCLC patients, of which 8786 males and 8868 females. Among the patients, 5512 $(31.22 \%)$ were treated neither with surgery or radiotherapy, $7184(40.69 \%)$ received surgery only, 652 (3.69\%) were given preoperative radiotherapy, 4206 (23.82\%) were given PORT, and $100(0.57 \%)$ were treated with radiotherapy both before and after surgery. The demographic and clinical parameter of patients are listed in Table 1. The tumor size and age were discretized and segmentation points were generated according to TNM stages (T1: tumor $\leq 3 \mathrm{~cm} \mathrm{T2:} \mathrm{tumor}>3 \mathrm{~cm}$ and $\leq 5 \mathrm{~cm}$ ) and by Youden index maximization, respectively.

There were statistically significant differences in all the baseline parameters between groups $(p<0.001)$. Patients that underwent surgery only $(40.69 \%)$ constitute the vast 


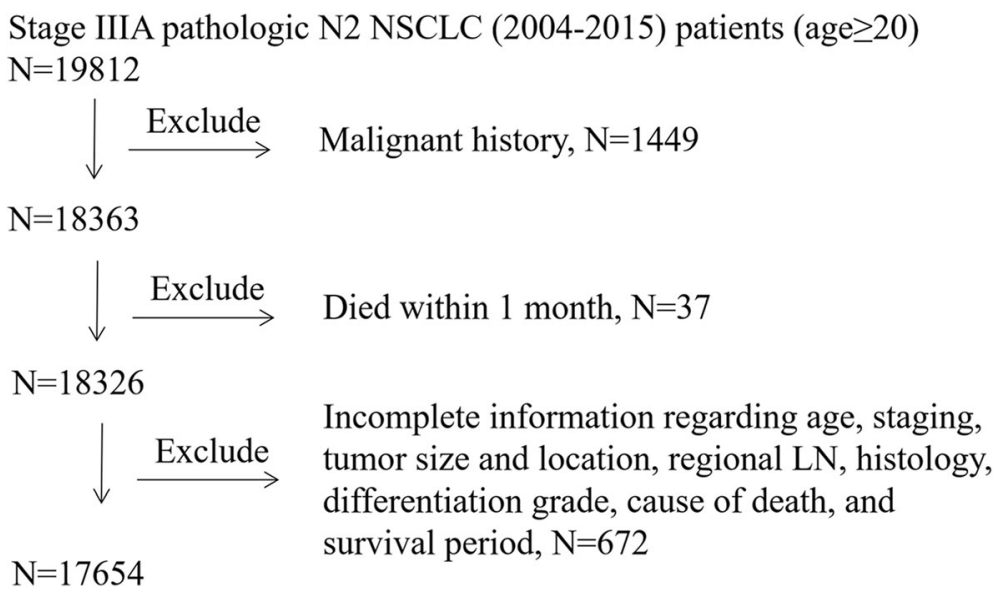

Fig. 1 Patient selection for this study

majority of patients included in the study, while those treated with radiotherapy both before and after surgery $(0.57 \%)$ were the least representative, especially in elderly patients. PORT with surgery constituted an increasing proportion of therapeutic procedures during the period considered (26.27\% from 2004 to 2007, 31.53\% from 2008 to 2011, and $42.20 \%$ from 2012 to 2017), whereas preoperative radiotherapy with surgery decreased (36.96\% from 2004 to 2007, 33.13\% from 2008 to 2011, and $29.91 \%$ from 2012 to 2017) in the same period (Fig. 2), which means during this period, PORT became more and more popular than preoperative radiotherapy. The majority of patients over 75 years of age refused radiotherapy $(84.83 \%)$ regardless of whether they underwent surgery, while the refusal rate was only at $67.43 \%$ in patients younger than 75 years. Additionally, patients who underwent radiotherapy combined with surgery (over 90\%) were more likely to receive chemotherapy than those who only had surgery (44.32\%). Moreover, patients who refused radiotherapy were older $(30.43 \%$ vs $2.32 \%$ over 75 years old) and less treated with chemotherapy than others (58.33\% vs $91.13 \%)$. Insured, married, high median household income people were more likely to have surgery and surgery combined with radiotherapy than uninsured, unmarried, and lower median household income people.

\section{Univariate and multivariate analysis}

In the univariate COX regression analysis of OS and LCSS, compared with patients underwent surgery only, the hazard ratio (HR), 95\% confidence interval (CI) $[\mathrm{HR}(95 \% \mathrm{CI})]$ of patients that underwent preoperative radiotherapy was $0.477(0.429-0.531)$ and $0.507(0.452-$ 0.568 ) for OS and LCSS, respectively. That of PORT patients was $0.632(0.602-0.662)$ and $0.645(0.612-0.679)$; of patients that underwent radiotherapy both before and after surgery was $0.593(0.466-0.755)$ and $0.56(0.426-$
0.736), and of patients that underwent neither radiotherapy nor surgery was $1.052(1.010-1.095)$ and 1.057(1.011$1.104)$. All the $p$-values were less than 0.05 . This analysis also showed that the following parameters are associated with a significantly shorter OS and LCSS: female sex, old age, not $\mathrm{AD}$, no chemotherapy, larger tumor, higher grade, no surgery or radiotherapy, white ethnicity, earlier year of diagnosis, non-upper lobe primary lesion, higher grade, unmarried, low income.

According to the multivariate analysis, age, sex, tumor size, histology, laterality, primary site, pathologic differentiation grade, chemotherapy and radiotherapy with surgery variables were statistically significant $(p<0.001)$. The multivariate analysis showed that all the four combination of surgery and radiotherapy promoted a better survival than having neither surgery nor radiotherapy. Patients with only surgery were taken as the reference for the subsequent analysis. The HR ( $95 \% \mathrm{CI}, \mathrm{p})$ of patients that underwent preoperative radiotherapy was 0.589 (0.529-0.657), $p<0.001$ and $0.606(0.539-0.681)$, $p<0.001$ for OS and LCSS, respectively. For patients that underwent PORT was $0.775(0.737-0.816), p<$ 0.001 and $0.772(0.731-0.816), p<0.001$ and for patients that underwent radiotherapy both before and after surgery was $0.752(0.590-0.957), p=0.021$ and 0.687 (0.522-0.904), $p=0.007$. For patients who refused surgery or radiotherapy, $p$ values were not significant. Results of the univariate and multivariate Cox regression of prognostic factors for OS and LCSS in IIIA/N2 NSCLC patients are shown in Table 2.

\section{Survival outcomes}

The median follow-up for the whole cohort was 39 months for OS and 48 months for LCSS. The median follow-up for the surgery only, preoperative radiotherapy with surgery, PORT with surgery, radiotherapy both before and after surgery, and no surgery or radiotherapy 
Table 1 Demographic and Clinical Parameters of patients with IIIA/N2 NSCLC

\begin{tabular}{|c|c|c|c|c|c|c|}
\hline Characteristics & $\begin{array}{l}\text { No surgery or } \\
\text { Radiotherapy } \\
(n=5512)\end{array}$ & $\begin{array}{l}\text { Surgery only } \\
(n=7184)\end{array}$ & $\begin{array}{l}\text { Preoperative } \\
\text { radiotherapy } \\
(n=652)\end{array}$ & $\begin{array}{l}\text { Postoperative } \\
\text { radiotherapy } \\
\text { (PORT, } n=4206)\end{array}$ & $\begin{array}{l}\text { Radiotherapy both } \\
\text { before and after } \\
\text { surgery }(n=100)\end{array}$ & $P$ value for $X^{2}$ \\
\hline Gender & & & & & & $<0.001$ \\
\hline Male & 2572(46.66\%) & $3680(51.22 \%)$ & $329(50.46 \%)$ & 2152(51.17\%) & $53(53.00 \%)$ & \\
\hline Female & 2940(53.34\%) & $3504(48.78 \%)$ & $323(49.54 \%)$ & 2054(48.83\%) & $47(47.00 \%)$ & \\
\hline Age & & & & & & $<0.001$ \\
\hline$<=75$ & 3893(70.63\%) & $4940(68.76 \%)$ & 608(93.25\%) & $3567(84.81 \%)$ & $92(92.00 \%)$ & \\
\hline$>75$ & $1619(29.37 \%)$ & $2244(31.24 \%)$ & $44(6.75 \%)$ & 639(15.19\%) & 8(8.00\%) & \\
\hline Histologic type & & & & & & $<0.001$ \\
\hline Adenocarcinoma & $2081(37.75 \%)$ & $3831(53.33 \%)$ & $342(52.45 \%)$ & $2486(59.11 \%)$ & $65(65.00 \%)$ & \\
\hline Squamous & 2118(38.43\%) & 1922(26.75\%) & $173(26.53 \%)$ & 1003(23.85\%) & 19(19.00\%) & \\
\hline Adenosquamous & $67(1.22 \%)$ & $158(2.20 \%)$ & $17(2.61 \%)$ & $88(2.09 \%)$ & $1(1.00 \%)$ & \\
\hline Large cell & $144(2.61 \%)$ & $201(2.80 \%)$ & $23(3.53 \%)$ & 129(3.07\%) & $1(1.00 \%)$ & \\
\hline OTHER & $1102(19.99 \%)$ & $1072(14.92 \%)$ & $97(14.88 \%)$ & $500(11.89 \%)$ & $14(14.00 \%)$ & \\
\hline Tumor Size $(\mathrm{cm})$ & & & & & & $<0.001$ \\
\hline$\leq 3$ & 2318(42.05\%) & $3765(52.41 \%)$ & 295(45.25\%) & 2483(59.03\%) & 47(47.00\%) & \\
\hline $3-5$ & $3194(57.95 \%)$ & $3419(47.59 \%)$ & $357(54.85 \%)$ & 1723(40.97\%) & $53(53.00 \%)$ & \\
\hline Race & & & & & & $<0.001$ \\
\hline White & 4444(80.62\%) & $5810(80.87 \%)$ & $544(83.44 \%)$ & $3436(81.69 \%)$ & 78(78.00\%) & \\
\hline Black & $763(13.84 \%)$ & $802(11.16 \%)$ & $68(10.43 \%)$ & $482(11.46 \%)$ & $9(9.00 \%)$ & \\
\hline Others & 297(5.39\%) & $553(7.70 \%)$ & 40(6.13\%) & $281(6.68 \%)$ & $13(13.00 \%)$ & \\
\hline Unknown & $8(0.15 \%)$ & $19(0.27 \%)$ & $0(0.00 \%)$ & $7(0.17 \%)$ & $0(0.00 \%)$ & \\
\hline Primary Site & & & & & & $<0.001$ \\
\hline Upper lobe & $3454(62.66 \%)$ & 4302(59.88\%) & $447(68.56 \%)$ & $2628(62.48 \%)$ & $65(65.00 \%)$ & \\
\hline Middle lobe & $241(4.37 \%)$ & $326(4.54 \%)$ & $34(5.21 \%)$ & $226(5.37 \%)$ & $5(5.00 \%)$ & \\
\hline Lower lobe & 1337(24.26\%) & $2141(29.80 \%)$ & 138(21.17\%) & 1098(26.11\%) & $27(27.00 \%)$ & \\
\hline NOS & $155(2.81 \%)$ & $195(2.71 \%)$ & $9(1.38 \%)$ & $123(2.92 \%)$ & $0(0.00 \%)$ & \\
\hline Overlapping lesion & $33(0.60 \%)$ & $64(0.89 \%)$ & $6(0.92 \%)$ & $28(0.67 \%)$ & $2(2.00 \%)$ & \\
\hline Main bronchus & $292(5.30 \%)$ & $156(2.17 \%)$ & $18(2.76 \%)$ & $103(2.45 \%)$ & $1(1.00 \%)$ & \\
\hline Grade & & & & & & $<0.001$ \\
\hline Grade I & $154(2.79 \%)$ & $327(4.55 \%)$ & $23(3.53 \%)$ & 138(3.28\%) & $3(3.00 \%)$ & \\
\hline Grade II & $900(16.33 \%)$ & 1935(26.93\%) & $138(21.17 \%)$ & $1021(24.27 \%)$ & $26(26.00 \%)$ & \\
\hline Grade III & $1722(31.24 \%)$ & $2427(33.78 \%)$ & $278(42.64 \%)$ & 1361(32.36\%) & $39(39.00 \%)$ & \\
\hline Grade IV & $83(1.51 \%)$ & $122(1.70 \%)$ & $5(0.77 \%)$ & $62(1.47 \%)$ & $3(3.00 \%)$ & \\
\hline unknown & 2653(48.13\%) & 2373(33.03\%) & 208(31.90\%) & 1624(38.61\%) & $29(29.00 \%)$ & \\
\hline Laterality & & & & & & 0.001 \\
\hline Right-origin of primary & $3421(62.06 \%)$ & $4316(60.08 \%)$ & $405(62.12 \%)$ & $2631(62.55 \%)$ & $67(67.00 \%)$ & \\
\hline Left-origin of primary & 2063(37.43\%) & $2840(39.53 \%)$ & $246(37.73 \%)$ & $1531(36.40 \%)$ & $33(33.00 \%)$ & \\
\hline Paired sit & $23(0.42 \%)$ & $20(0.28 \%)$ & $1(0.15 \%)$ & $38(0.90 \%)$ & $0(0.00 \%)$ & \\
\hline Only one side - side unspecified & $4(0.07 \%)$ & $6(0.08 \%)$ & $0(0.00 \%)$ & $4(0.10 \%)$ & $0(0.00 \%)$ & \\
\hline Not a paired site & $1(0.02 \%)$ & $2(0.03 \%)$ & $0(0.00 \%)$ & $2(0.05 \%)$ & $0(0.00 \%)$ & \\
\hline Insurance status & & & & & & $<0.001$ \\
\hline Medicaid & $594(10.78 \%)$ & $768(10.70 \%)$ & $45(6.90 \%)$ & 399(9.49\%) & $7(7.00 \%)$ & \\
\hline Uninsured & 101(1.83\%) & $118(1.65 \%)$ & $10(1.53 \%)$ & $59(1.40 \%)$ & $2(2.00 \%)$ & \\
\hline Unknown & $1316(23.88 \%)$ & $1858(25.86 \%)$ & $180(27.61 \%)$ & $847(20.14 \%)$ & $29(29.00 \%)$ & \\
\hline
\end{tabular}


Table 1 Demographic and Clinical Parameters of patients with IIIA/N2 NSCLC (Continued)

\begin{tabular}{|c|c|c|c|c|c|c|}
\hline Characteristics & $\begin{array}{l}\text { No surgery or } \\
\text { Radiotherapy } \\
(n=5512)\end{array}$ & $\begin{array}{l}\text { Surgery only } \\
(n=7184)\end{array}$ & $\begin{array}{l}\text { Preoperative } \\
\text { radiotherapy } \\
(n=652)\end{array}$ & $\begin{array}{l}\text { Postoperative } \\
\text { radiotherapy } \\
\text { (PORT, } n=4206)\end{array}$ & $\begin{array}{l}\text { Radiotherapy both } \\
\text { before and after } \\
\text { surgery }(n=100)\end{array}$ & $P$ value for $X^{2}$ \\
\hline Insured & $3501(63.52)$ & $4440(61.80 \%)$ & $417(63.96 \%)$ & $2901(68.97 \%)$ & $62(62.00 \%)$ & \\
\hline Marital status & & & & & & $<0.001$ \\
\hline Married & $2812(51.02 \%)$ & $3644(50.72 \%)$ & $393(60.28 \%)$ & $2440(58.01 \%)$ & $58(58.00 \%)$ & \\
\hline Single & $671(12.17 \%)$ & $887(12.35 \%)$ & $76(11.66 \%)$ & $467(11.10 \%)$ & $12(12.00 \%)$ & \\
\hline Divorced & $681(12.35 \%)$ & $903(12.57 \%)$ & $93(14.26 \%)$ & $540(12.84 \%)$ & $12(12.00 \%)$ & \\
\hline widowed & 1079(19.6\%) & $1401(19.50 \%)$ & $63(9.66 \%)$ & $574(13.65 \%)$ & $11(11.00 \%)$ & \\
\hline Unknown & $181(3.28 \%)$ & $262(3.65 \%)$ & $20(3.07 \%)$ & 131(3.11\%) & $1(1.00 \%)$ & \\
\hline Unmarried or domestic partner & $6(0.11 \%)$ & $12(0.17 \%)$ & $1(0.15 \%)$ & $12(0.29 \%)$ & $0(0.00 \%)$ & \\
\hline Separated & $82(1.49 \%)$ & $75(1.04 \%)$ & $6(0.92 \%)$ & $42(1.00 \%)$ & $6(6.00 \%)$ & \\
\hline Year of diagnosis & & & & & & $<0.001$ \\
\hline $2004-2007$ & $1756(31.86 \%)$ & 2399(33.39\%) & $241(36.96 \%)$ & $1105(26.27 \%)$ & $37(37.00 \%)$ & \\
\hline 2008-2011 & 1982(35.96\%) & $2437(33.92 \%)$ & $216(33.13 \%)$ & $1326(31.53 \%)$ & $35(35.00 \%)$ & \\
\hline $2012-2017$ & 1774(32.18\%) & 2348(32.68\%) & 195(29.91\%) & $1775(42.20 \%)$ & $28(28.00 \%)$ & \\
\hline Region & & & & & & $<0.001$ \\
\hline EAST & 2733(49.58\%) & $3243(45.14 \%)$ & $283(43.40 \%)$ & $2148(51.07 \%)$ & $38(38.00 \%)$ & \\
\hline NORTHWEST & 1965(35.65\%) & $3082(42.90 \%)$ & $265(40.64 \%)$ & 1417(33.69\%) & $50(50.00 \%)$ & \\
\hline SOUTHWEST & $661(11.99 \%)$ & $647(9.01 \%)$ & $86(13.19 \%)$ & $561(13.34 \%)$ & $10(10.00 \%)$ & \\
\hline Pacific Coast & $153(2.78 \%)$ & $212(2.95 \%)$ & $18(2.76 \%)$ & $80(1.90 \%)$ & $2(2.00 \%)$ & \\
\hline High school education & & & & & & $<0.001$ \\
\hline$\geq 21$ & $977(17.72 \%)$ & 1524(21.21\%) & $102(15.64 \%)$ & $590(14.03 \%)$ & $19(19.00 \%)$ & \\
\hline $13-20$ & 1823(33.07\%) & 2293(31.92\%) & 159(24.39\%) & 1199(28.51\%) & $32(32.00 \%)$ & \\
\hline $7-12.99$ & $2363(42.87 \%)$ & 2968(41.31\%) & $344(52.76 \%)$ & $2101(49.95 \%)$ & $41(41.00 \%)$ & \\
\hline$<7$ & $349(6.33 \%)$ & $399(5.55 \%)$ & $47(7.21 \%)$ & $316(7.51 \%)$ & $8(8.00 \%)$ & \\
\hline Median household income, in tens & & & & & & $<0.001$ \\
\hline$<38,000$ & $422(7.66 \%)$ & $570(7.927 \%)$ & $28(4.29 \%)$ & $290(6.89 \%)$ & $5(5.00 \%)$ & \\
\hline $38,000-47,999$ & 1080(19.59\%) & 1097(15.27\%) & $88(13.50 \%)$ & $731(17.38 \%)$ & 14(14.00\%) & \\
\hline $48,000-62,999$ & 2064(37.45\%) & 2842(39.56\%) & $276(42.33 \%)$ & 1588(37.76\%) & $40(40.00 \%)$ & \\
\hline$>63,000$ & 1946(35.30\%) & 2675(37.24\%) & 260(39.88\%) & 1597(37.97\%) & $41(41.00 \%)$ & \\
\hline Chemotherapy & & & & & & $<0.001$ \\
\hline Yes & $4221(76.58 \%)$ & $3184(44.32 \%)$ & $631(96.78 \%)$ & $3791(90.13 \%)$ & 96(96.00\%) & \\
\hline no & $1291(23.42 \%)$ & $4000(55.68 \%)$ & $21(3.22 \%)$ & 415(9.87\%) & $4(4.00 \%)$ & \\
\hline
\end{tabular}

Abbreviations: NSCLC Non-small cell lung cancer, IIIA/N2 Stage IIIA pathologic N2

groups were $36,66,51,55$ and 31 months, respectively for OS and 45, 72, 59, 66 and 40 months for LCSS. Patients who received preoperative radiotherapy with surgery had the longest 5-year overall survival (42.84\%) and lung cancer-specific survival (47.12\%).

The Kaplan-Meier method was used to estimate the OS and LCSS, showing that preoperative radiotherapy was the optimal strategy among IIIA/N2 patients $(p<$ $0.001)$. Moreover, patients that underwent surgery had better survival than who refused it $(p<0.001)$. The survival of patients that underwent surgery combined with radiotherapy was better than patients who underwent surgery only $(p<0.001)$. The Survival analysis was performed using the log-rank test, and showed that the pairwise difference between each groups were statistically significant (Fig. 3).

Similar results were observed in the subgroups (sex, age, tumor size, primary site, chemotherapy, histology, pathologic differentiation grade) analysis of the OS and LCSS Kaplan-Meier. Importantly, considering that most patients with IIIA/N2 NSCLC received chemotherapy in clinical, the chemotherapy subgroup was the most practical one. In chemotherapy subgroup, there were almost the same survival curves as overall survival curves and 


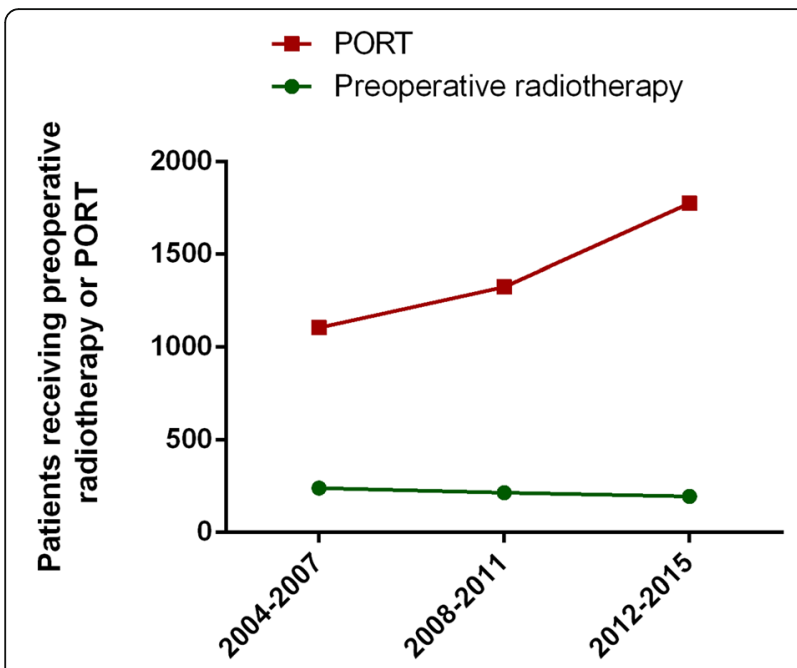

Fig. 2 Number of IIIA/N2 NSCLC patients underwent preoperative radiotherapy and PORT from 2004 to 2015. There were 241, 216, 195 IIIA/N2 NSCLC patients received preoperative radiotherapy with surgery in 2004-2007, 2008-2011 and 2012-2017, respectively. Whereas, the number of IIIA/N2 NSCLC patients who underwent PORT with surgery were $1105,1326,1775$ in the same time periods as above. Abbreviations: NSCLC, non-small cell lung cancer; IIIA/N2, stage IIIA pathologic N2; PORT, postoperative radiotherapy

the survival rate of preoperative radiotherapy of both OS and LCSS were the highest in this subgroup. Interestingly, we also found that the optimal treatment for the subgroup of patients with $>75$ years old was PORT, while in the subgroup of no chemotherapy the optimal treatment may be radiotherapy both before and after surgery (Fig. 4, Fig. 5). The OS and LCSS analysis showed that the survival rate of patients that underwent preoperative radiotherapy was not significantly different with patients who underwent PORT in the AD subgroup ( $p=0.8274$ and 0.7653 for OS and LCSS analysis, respectively). Moreover, there was no significant difference between the survival of patients who refused surgery and that of patients who received surgery only $(p=0.6848$ and 0.5293 for OS and LCSS analysis, respectively) in the subgroup of patients with age $>75$. Thus, the quality of life may be the main consideration for IIIA/N2 NSCL $C$ patients with age $>75$.

\section{Discussion}

In this study, we used the SEER Database to analyze the prognostic value of surgery and radiotherapy combinations and the relationship between therapeutic strategy with survival and HRs in IIIA/N2 NSCLC patients. Our data demonstrated that the use of surgery in IIIA/N2 NSCLC patients was effective in all the analyzed groups, except in patients older than 75 years old. In subgroup of age $>75$, surgery did not improve survival, and PORT was more recommended if having surgery and radiation. For patients who underwent surgery combined with radiotherapy, the preoperative radiotherapy regimen led to the best result, while those who underwent neither surgery nor radiotherapy had the worst prognosis. Clinically, since adjuvant chemotherapy has become a standard treatment, the results of the chemotherapy subgroup deserve special attention, which were generally consistent with the overall OS and LCSS. The LCSS rate in the overall patients was similar to of the OS rate. In the Cox-regression analysis, the following parameters were associated with a higher risk of death: female sex, age > 75, SCC and other histologic types, no chemotherapy, poor differentiation, and larger tumor. With so many high-risk parameters, the results of survival analysis in subgroups were of great significance and we will further analyze the influence of age, gender, histologic types, chemotherapy, pathologic differentiation grade and tumor size on the survival of IIIA/N2 NSCLC patients in the future.

There was significant heterogeneity in the survival rate of IIIA/N2 NSCLC patients, for which the standard treatment had been debated for a long time [11, 21-23]. Although the optimal treatment approach in IIIA/N2 NSCLC patients remains undetermined, almost all studies confirmed the effectiveness of surgery. A recent review argued that in patients with IIIA/N2 NSCLC, radical resection with lymph node dissection is reasonable and early operation leads to a greater benefit [24]. Bryan DS et al. found that surgery improve survival in IIIA/N2 NSCLC, and in a survey, most thoracic surgeons recommended surgery as part of the therapy for IIIA/N2 NSCLC patients, because resection improves the rate of local control [25]. Consistently with this view, patients younger than 75 years old who underwent surgery showed an improved survival rate compared to those that do not in our study. However, there is still a lack of high-quality prospective evidence showing that how the different surgical procedures influence the survival rates.

Historically, surgery alone or combined with chemotherapy and radiotherapy has been the most common approach, and in recent decades there is a trend of performing radiotherapy before surgery. Previous studies reported that radiotherapy followed by surgery gave a survival benefit for IIIA/N2 NSCLC patients [26-29]. Several trials have been performed to determine the safety and efficacy of the combination of chemo- and radiotherapy [13, 27, 30-34]. Some of those reports demonstrated that chemotherapy combined with radiotherapy prolonged the survival of III/N2 NSCLC patients, as we found in this study $[27,30]$ while one clinical trial showed that radiotherapy combined with chemotherapy does not improve survival of IIIA/N2 NSCLC patients that underwent surgery [33]. Another study [35] suggested that surgery combined with chemoradiotherapy could improve the 
Table 2 Multivariate COX hazards regression for OS and LCSS in IIIA/N2 NSCLC patients based on prognostic factors

\begin{tabular}{|c|c|c|c|c|c|c|c|c|}
\hline \multirow[t]{3}{*}{ Variables } & \multicolumn{4}{|l|}{ OS } & \multicolumn{4}{|l|}{ LCSS } \\
\hline & \multicolumn{2}{|l|}{ Univariate analysis } & \multicolumn{2}{|l|}{ Multivariate analysis } & \multicolumn{2}{|l|}{ Univariate analysis } & \multicolumn{2}{|l|}{ Multivariate analysis } \\
\hline & $\mathrm{HR}(95 \% \mathrm{Cl})$ & $\mathrm{P}$ & $\mathrm{HR}(95 \% \mathrm{Cl})$ & $P$ & $\mathrm{HR}(95 \% \mathrm{Cl})$ & $P$ & $\mathrm{HR}(95 \% \mathrm{Cl})$ & $\mathrm{P}$ \\
\hline Gender & & $<0.001$ & & $<0.001$ & & $<0.001$ & & $\overline{<0.001}$ \\
\hline Female & Reference & & Reference & & Reference & & Reference & \\
\hline Male & $0.799(0.772,0.828)$ & $<0.001$ & $0.823(0.794,0.853)$ & $<0.001$ & $0.823(0.792,0.855)$ & $<0.001$ & $0.845(0.813,0.879)$ & $<0.001$ \\
\hline Age & & $<0.001$ & & $<0.001$ & & $<0.001$ & & $<0.001$ \\
\hline$<75$ & Reference & & Reference & & Reference & & Reference & \\
\hline$>=75$ & $1.645(1.582,1.710)$ & $<0.001$ & $1.364(1.310,1.421)$ & $<0.001$ & $1.590(1.524,1.659)$ & $<0.001$ & $1.334(1.276,1.395)$ & $<0.001$ \\
\hline Histology & & $<0.001$ & & $<0.001$ & & $<0.001$ & & $<0.001$ \\
\hline Adenocarcinoma & Reference & & Reference & & Reference & & Reference & \\
\hline Squamous & $1.429(1.372,1.489)$ & $<0.001$ & $1.228(1.177,1.281)$ & $<0.001$ & $1.388(1.328,1.451)$ & $<0.001$ & $1.193(1.139,1.250)$ & $<0.001$ \\
\hline Adenosquamous & $1.159(1.018,1.319)$ & 0.026 & $1.114(0.977,1.269)$ & 0.106 & $1.138(0.988,1.312)$ & 0.073 & $1.087(0.943,1.254)$ & 0.25 \\
\hline Large cell & $1.248(1.125,1.384)$ & $<0.001$ & $1.159(1.041,1.292)$ & 0.007 & $1.303(1.167,1.456)$ & $<0.001$ & $1.209(1.077,1.357)$ & 0.001 \\
\hline OTHER & $1.395(1.328,1.464)$ & $<0.001$ & $1.187(1.128,1.250)$ & $<0.001$ & $1.411(1.339,1.488)$ & $<0.001$ & $1.196(1.132,1.265)$ & $<0.001$ \\
\hline Chemotherapy & & $<0.001$ & & $<0.001$ & & $<0.001$ & & $<0.001$ \\
\hline No & Reference & & Reference & & Reference & & Reference & \\
\hline Yes & $0.556(0.536,0.577)$ & $<0.001$ & $0.635(0.610,0.662)$ & $<0.001$ & $0.586(0.563,0.610)$ & $<0.001$ & $0.665(0.636,0.695)$ & $<0.001$ \\
\hline Tumor Size & & $<0.001$ & & $<0.001$ & & $<0.001$ & & $<0.001$ \\
\hline$\leq 3$ & Reference & & Reference & & Reference & & Reference & \\
\hline $3--5$ & $1.275(1.231,1.320)$ & $<0.001$ & $1.221(1.178,1.266)$ & $<0.001$ & $1.311(1.261,1.362)$ & $<0.001$ & $1.258(1.209,1.308)$ & $<0.001$ \\
\hline Grade & & $<0.001$ & & $<0.001$ & & $<0.001$ & & $<0.001$ \\
\hline Grade I & Reference & & Reference & & Reference & & Reference & \\
\hline Grade ॥ & $1.128(1.015,1.253)$ & 0.025 & $1.134(1.020,1.260)$ & 0.02 & $1.147(1.021,1.289)$ & 0.021 & $1.154(1.027,1.297)$ & 0.016 \\
\hline Grade III & $1.34(1.210,1.485)$ & $<0.001$ & $1.334(1.203,1.480)$ & $<0.001$ & $1.399(1.249,1.567)$ & $<0.001$ & $1.386(1.235,1.554)$ & $<0.001$ \\
\hline Grade IV & $1.593(1.351,1.880)$ & $<0.001$ & $1.512(1.275,1.794)$ & $<0.001$ & $1.604(1.336,1.926)$ & $<0.001$ & $1.495(1.237,1.806)$ & $<0.001$ \\
\hline unknow & $1.569(1.417,1.737)$ & $<0.001$ & $1.496(1.348,1.660)$ & $<0.001$ & $1.627(1.453,1.821)$ & $<0.001$ & $1.544(1.377,1.732)$ & $<0.001$ \\
\hline Radiation with surgery & & $<0.001$ & & $<0.001$ & & $<0.001$ & & $<0.001$ \\
\hline Only surgery & Reference & & Reference & & Reference & & Reference & \\
\hline Preoperative radiotherapy & $0.477(0.429,0.531)$ & $<0.001$ & $0.589(0.529,0.657)$ & $<0.001$ & $0.507(0.452,0.568)$ & $<0.001$ & $0.606(0.539,0.681)$ & $<0.001$ \\
\hline postoperative radiotherapy & $0.632(0.602,0.662)$ & $<0.001$ & $0.775(0.737,0.816)$ & $<0.001$ & $0.645(0.612,0.679)$ & $<0.001$ & $0.772(0.731,0.816)$ & $<0.001$ \\
\hline $\begin{array}{l}\text { Raiotherapy both before } \\
\text { and after sugery }\end{array}$ & $0.593(0.466,0.755)$ & $<0.001$ & $0.752(0.590,0.957)$ & 0.021 & $0.56(0.426,0.736)$ & $<0.001$ & $0.687(0.522,0.904)$ & 0.007 \\
\hline No surgery or radiation & $1.052(1.010,1.095)$ & 0.015 & $1.035(0.991,1.082)$ & 0.121 & $1.057(1.011,1.104)$ & 0.014 & $1.023(0.975,1.073)$ & 0.356 \\
\hline Primary Site & & $<0.001$ & & $<0.001$ & & $<0.001$ & & $<0.001$ \\
\hline Upper lobe & Reference & & Reference & & Reference & & Reference & \\
\hline Middle lobe & $1.026(0.943,1.116)$ & 0.557 & $1.009(0.926,1.099)$ & 0.833 & $1.053(0.962,1.153)$ & 0.26 & $1.026(0.935,1.124)$ & 0.592 \\
\hline Lower lobe & $1.103(1.059,1.149)$ & $<0.001$ & $1.08(1.037,1.125)$ & $<0.001$ & $1.102(1.054,1.151)$ & $<0.001$ & $1.078(1.032,1.127)$ & 0.001 \\
\hline NOS & $1.303(1.172,1.449)$ & $<0.001$ & $1.314(1.172,1.474)$ & $<0.001$ & $1.29(1.148,1.449)$ & $<0.001$ & $1.311(1.157,1.486)$ & $<0.001$ \\
\hline Overlapping lesion & $0.985(0.808,1.199)$ & 0.877 & $1.024(0.840,1.248)$ & 0.814 & $0.922(0.738,1.153)$ & 0.478 & $0.945(0.756,1.182)$ & 0.62 \\
\hline Main bronchus & $1.252(1.136,1.379)$ & $<0.001$ & $1.128(1.023,1.245)$ & 0.016 & $1.302(1.174,1.444)$ & $<0.001$ & $1.171(1.054,1.301)$ & 0.003 \\
\hline Laterality & & 0.001 & & $<0.001$ & & $<0.001$ & & $<0.001$ \\
\hline Right-origin of primary & Reference & & Reference & & Reference & & Reference & \\
\hline Left-origin of primary & $0.932(0.899,0.966)$ & $<0.001$ & $0.926(0.893,0.961)$ & $<0.001$ & $0.907(0.872,0.944)$ & $<0.001$ & $0.904(0.868,0.941)$ & $<0.001$ \\
\hline Paired sit & $0.836(0.633,1.104)$ & 0.206 & $0.717(0.532,0.966)$ & 0.029 & $0.809(0.595,1.100)$ & 0.177 & $0.694(0.500,0.964)$ & 0.029 \\
\hline $\begin{array}{l}\text { Only one side - side } \\
\text { unspecified }\end{array}$ & $1.556(0.861,2.810)$ & 0.143 & $1.339(0.737,2.430)$ & 0.338 & $1.14(0.543,2.391)$ & 0.73 & $0.991(0.470,2.090)$ & 0.982 \\
\hline Not a paired site & $1.266(0.408,3.927)$ & 0.683 & $1.371(0.438,4.288)$ & 0.587 & $1.532(0.494,4.752)$ & 0.46 & $1.647(0.526,5.158)$ & 0.392 \\
\hline
\end{tabular}




\section{Overall Survival}

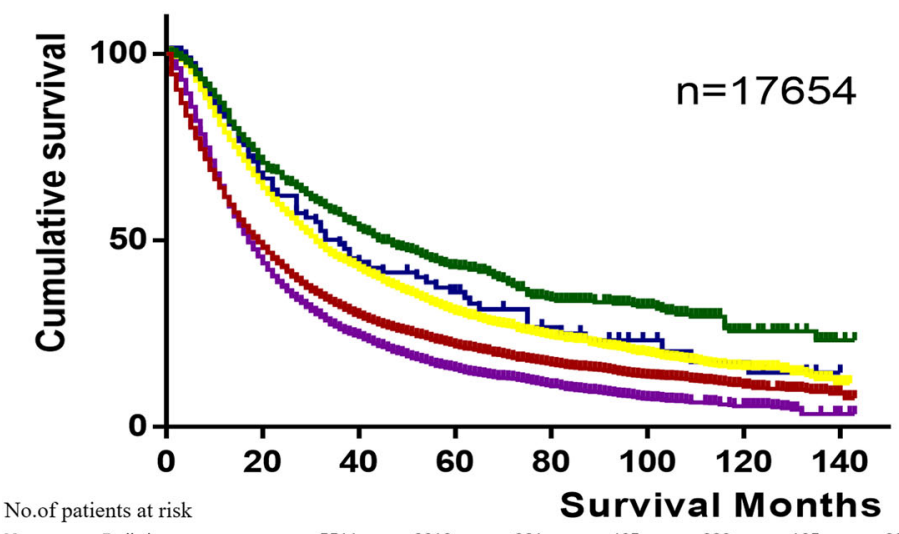

$\begin{array}{lccccccc}\text { No surgery or Radiation } & 5511 & 2019 & 921 & 465 & 239 & 105 & 33 \\ \text { Surgery only } & 7183 & 2763 & 1448 & 846 & 501 & 280 & 103 \\ \text { Preoperative radiotherapy } & 651 & 404 & 255 & 166 & 107 & 73 & 27 \\ \text { postoperative radiotherapy } & 4205 & 2131 & 1107 & 601 & 329 & 172 & 71 \\ \text { Radiation both before and after surgery } & 99 & 58 & 35 & 25 & 16 & 9 & 6\end{array}$

\section{Lung cancer specific survival}

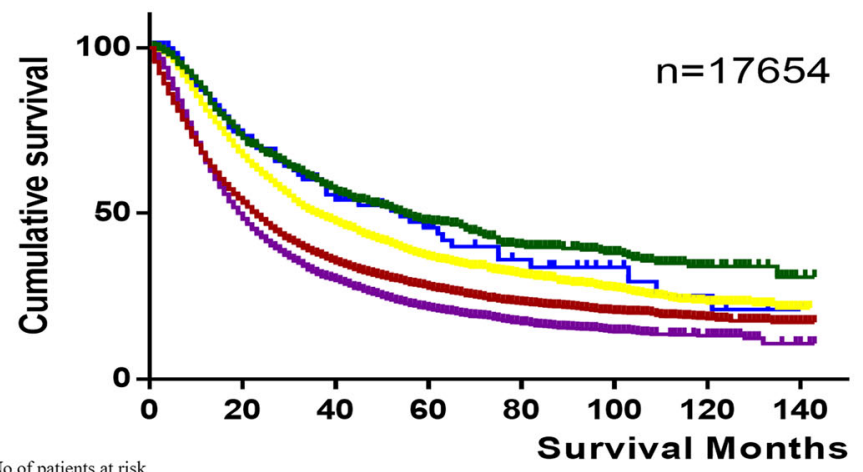

$\begin{array}{lcccccccc}\text { No.of patients at risk } & & & & & & & & \\ \text { No surgery or Radiation } & 511 & 2131 & 1107 & 601 & 329 & 172 & 71 & 6 \\ \text { Surgery only } & 7183 & 2762 & 1448 & 846 & 501 & 280 & 103 & 7 \\ \text { Preoperative radiotherapy } & 651 & 405 & 255 & 166 & 107 & 73 & 27 & 3 \\ \text { postoperative radiotherapy } & 4205 & 2131 & 1107 & 601 & 329 & 172 & 71 & 6 \\ \text { Radiation both before and after surgery } & 99 & 58 & 35 & 25 & 16 & 9 & 6 & 0\end{array}$

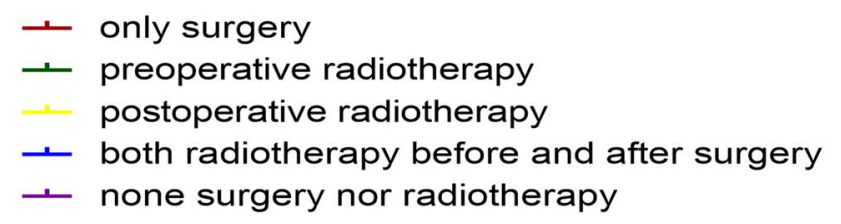

Fig. 3 Kaplan-Meier analysis of different radiotherapy sequences on OS and LCSS of IIIA/N2 NSCLC patients. It showed that both in OS and LCSS of IIIA/N2 NSCLC patients, preoperative radiotherapy was the best strategy, and then were both preoperative and PORT, PORT, only surgery and neither surgery nor radiotherapy. Log Rank $p<0.001$. The number of patients at risk in different time periods was under survival curves. Abbreviations: NSCLC, non-small cell lung cancer; IIIA/N2, stage IIIA pathologic N2; OS, Overall survival; LCSS, Lung cancer specific survival

patients' survival. Therefore, in previous studies there is no consensus for the optimal combination time of radiotherapy and chemotherapy [36]. In our study, we demonstrated that preoperative radiotherapy is the best strategy for approaches combining chemo and radiotherapy, consistently with a previous study based on SEER data [17], as well as another independent study [13].
The subgroup of patients with an age $>75$ was different from the others, because surgery only did not appear to significantly improve survival in comparison to no surgery. PORT was the optimal treatment strategy in cases having surgery. These results can be explained by a worse treatment tolerance in this subgroup. In addition to that, these patients also underwent radiotherapy less frequently and 


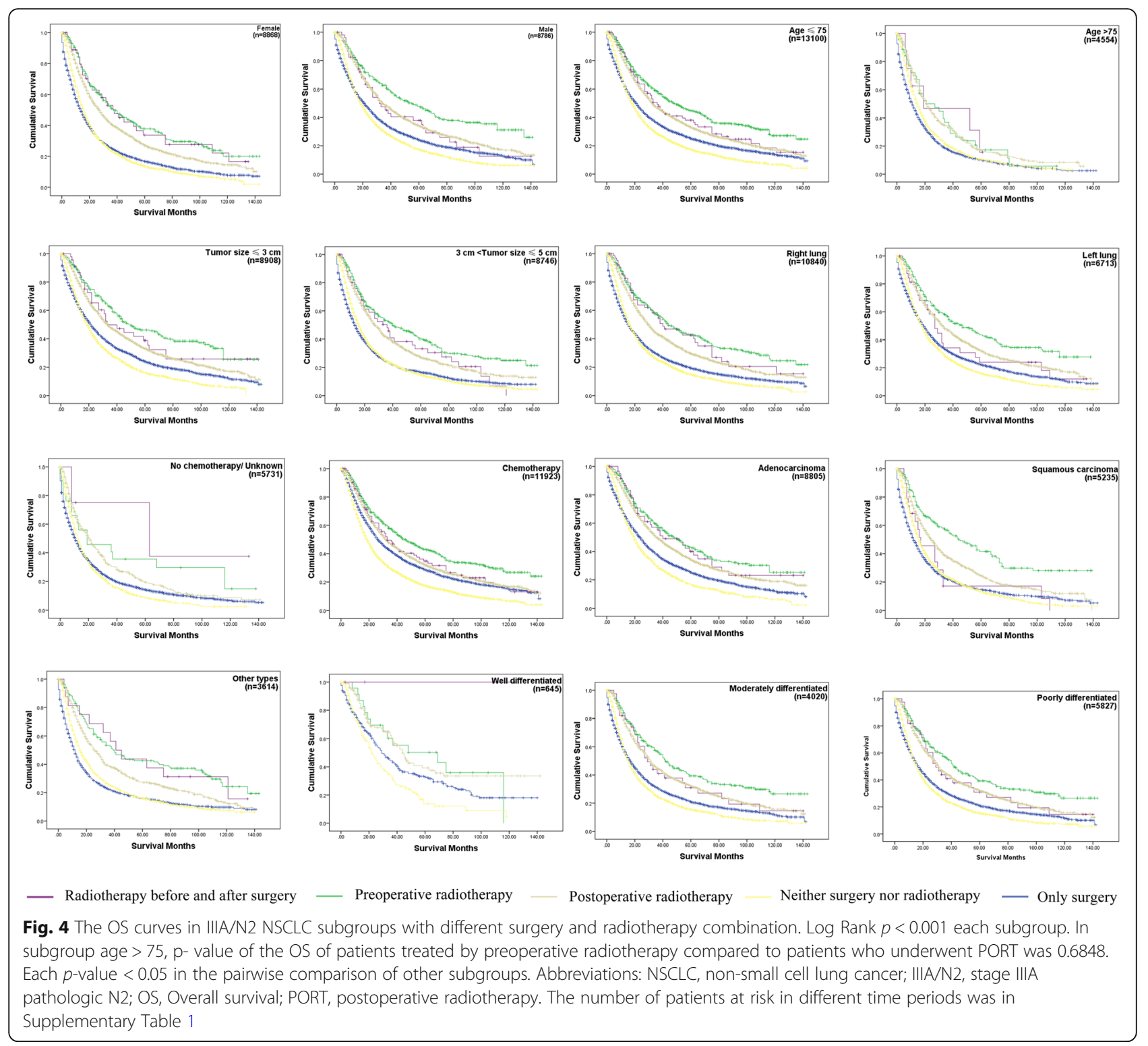

had a higher death risk, consistently to a previous study [37]. Therefore, it is important to determine optimal treatment for the elderly population. However, older and frail patients are often excluded from clinical trials using strict eligibility criteria [38] and receive less standard treatment $[39,40]$. Thus, future research should focus on the elderly population who could benefit from specific treatment regimens.

The SEER database includes a population size larger than other clinical trials [41], and the inclusion of the revision made by the TNM classification project makes it more reliable to predict the survival outcomes [42-44]. This study has several limitations: other than the intrinsic defects of retrospective study, important information, such as gene mutations, lymph nodes station, chemotherapy sequences, and extent of resection are not provided in the SEER database. Moreover, patients treated with radiotherapy are more likely to be treated with chemotherapy as well. As role of chemotherapy must be seriously taken into account [30], this study could have a bias favoring radiotherapy. Future prospective studies will need more detailed information on chemotherapy data.

\section{Conclusions}

According to this study, the different clinical outcomes between different strategies of surgery and radiotherapy combination may be useful to determine prognosis and to provide an important reference for clinicians and patients. Our data revealed that surgery, radiotherapy and chemotherapy were associated with improved OS and LCSS in patients with IIIA/N2 NSCLC. Younger patients 


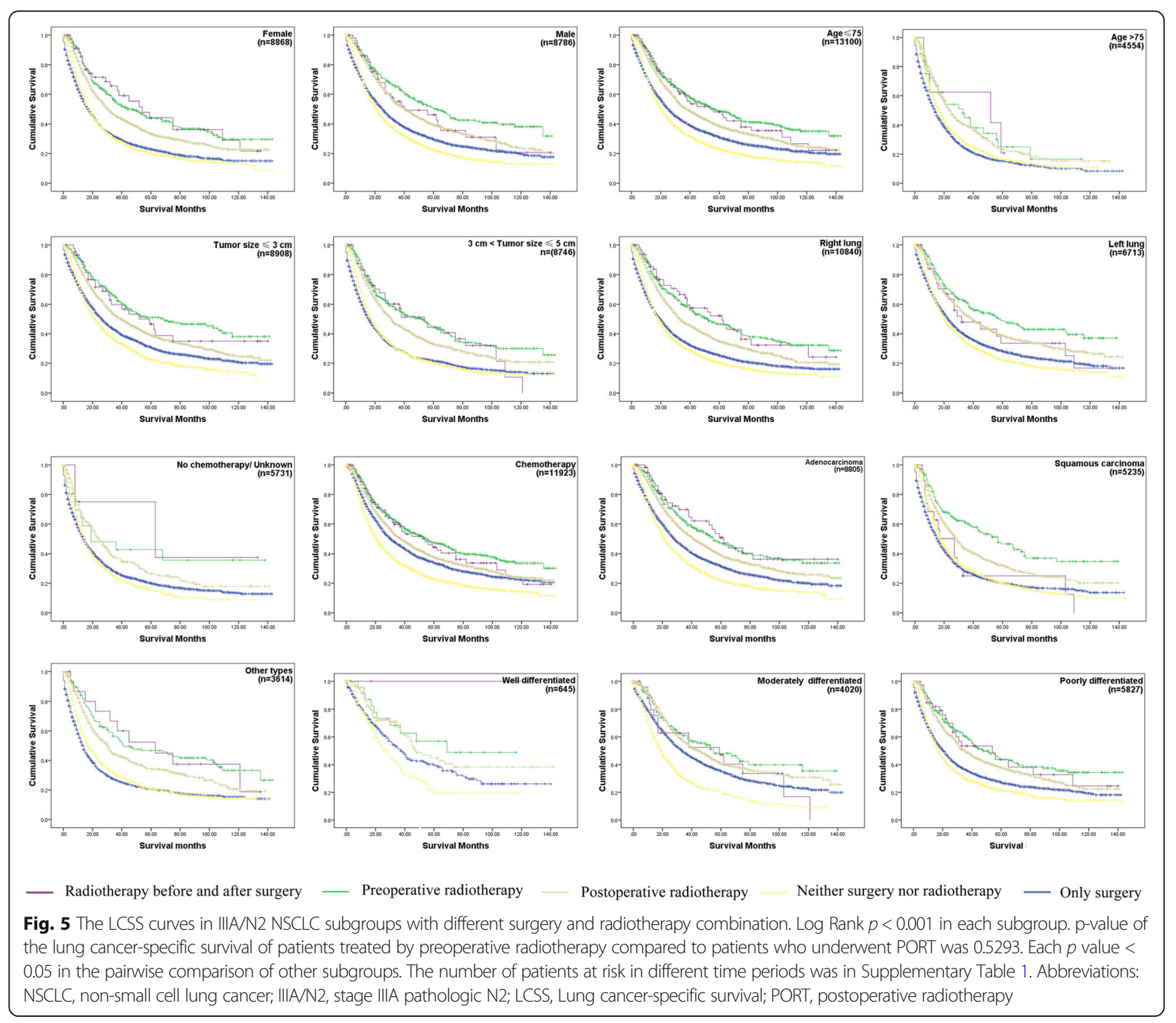

underwent surgical resection and preoperative radiotherapy had the best chance of survival $(p<0.001)$. Based on our results, the rank of therapeutic strategies to improve OS and LCSS in IIIA/N2 NSCLC patients is the following: preoperative radiotherapy $>$ radiotherapy both before and after surgery (considering the small number of people in this subgroup, we do not make a positive recommendation for this sequence, but this method should not be rejected or excluded, it worth more detailed relevant research in the future) $>$ PORT $>$ only surgery $>$ neither surgery nor radiotherapy $(p<0.001)$. In conclusion, preoperative radiotherapy is worth attention. However, in patients with more than 75 years, there was no clear benefit from surgery. The quality of life should be the main consideration for them when making choice. Novel treatments like immunotherapy and targeting therapy as additional options will need to be evaluated in future studies.

\section{Supplementary information}

Supplementary information accompanies this paper at https://doi.org/10. 1186/s12885-020-07309-y.

Additional file 1: Table S1. The number of patients at risk in different time periods in different subgroups.

\section{Abbreviations}

NSCLC: Non-small cell lung cancer; IIIA/N2: Stage IIIA pathologic N2; OS: Overall survival; LCSS: Lung cancer-specific survival; SEER: Surveillance, Epidemiology, and End Results registry; HRs: Hazard ratios;

PORT: Postoperative radiotherapy; AD: Adenocarcinoma; SQCC: Squamous cell carcinoma; LCC: Large cell carcinoma

\section{Acknowledgments}

We would like to thank all the staff of the National Cancer Institute for their efforts in the SEER program.

\section{Authors' contributions}

Guarantor of integrity of the entire study H.X.D, S.S.X, C.H.W. Study concepts and design H.X.D, S.S.X. Literature research H.X.D, S.S.X. Clinical studies S.S.X, L.L. Experimental studies / data analysis H.X.D. Statistical analysis H.X.D, L.L, 
S.S.X. Manuscript preparation H.X.D, S.S.X, C.H.W. Manuscript editing S.S.X, C.H.W. All authors had read and approved the manuscript.

\section{Funding}

This work was supported by the National Natural Science Foundation of China (No. 81802262). We also acknowledge the financial support of the Fundamental Research Funds for the Central Universities (No. 22120180584). Changhui Wang is the recipient of the foundations and was responsible for designing, guaranteeing of integrity of the entire study, editing manuscript in our study.

\section{Availability of data and materials}

All the data in the article was from SEER database at http://seer.cancer.gov/., which contain data from 18 registered cancer institute covering nearly $26 \%$ of the total US population.

\section{Ethics approval and consent to participate}

This research including formal consent was exempt by the ethics committee of the 10th People's Hospital affiliated to Tongji University. All the data in the article was from SEER database, which was publicly available, and we received permission for using the data on non-commercial use.

\section{Consent for publication}

Not Applicable.

\section{Competing interests}

The authors declare no potential conflicts of interest related to this study.

Received: 15 April 2020 Accepted: 17 August 2020

Published online: 26 August 2020

\section{References}

1. Siegel R, Ma J, Zou Z, Jemal A. Cancer statistics, 2014. CA Cancer J Clin. 2014;64(1):9-29. https://doi.org/10.3322/caac.21208.

2. Mountain CF. Staging classification of lung cancer. A critical evaluation. Clin Chest Med. 2002;23(1):103-21.

3. Sawabata N, Miyaoka E, Asamura H, Nakanishi Y, Eguchi K, Mori M, Nomori H, Fujii Y, Okumura M, Yokoi K, R. Japanese Joint Committee for Lung Cancer. Japanese lung cancer registry study of 11,663 surgical cases in 2004: demographic and prognosis changes over decade. J Thorac Oncol. 2011; 6(7):1229-35. https://doi.org/10.1097/JTO.0b013e318219aae2.

4. Siegel RL, Miller KD, Jemal A. Cancer statistics, 2017. CA Cancer J Clin. 2017; 67(1):7-30. https://doi.org/10.3322/caac.21387.

5. Zeng X, Karnon J, Wang S, Wu B, Wan X, Peng L. The cost of treating advanced non-small cell lung cancer: estimates from the chinese experience. PLoS One. 2012;7(10):e48323. https://doi.org/10.1371/journal. pone.0048323.

6. Goldstraw P, Crowley J, Chansky K, Giroux DJ, Groome PA, Rami-Porta R, Postmus PE, Rusch V, Sobin L, C. International Association for the Study of Lung Cancer International Staging, and I. Participating. The IASLC lung Cancer staging project: proposals for the revision of the TNM stage groupings in the forthcoming (seventh) edition of the TNM classification of malignant tumours. J Thorac Oncol. 2007;2(8):706-14. https://doi.org/10. 1097/JTO.0b013e31812f3c1a.

7. Shi Y, Sun Y, Yu J, Ding C, Wang Z, Wang C, Wang D, Wang C, Wang Z, Wang M, Zhi X, Lu Y, Feng J, Liu Y, Liu X, Liu W, Wu G, Li X, Li K, Li E, Li W, Chen G, Chen Z, Yu P, Wu N, Wu M, Xiao W, Zhang L, Zhang Y, Zhang S, Yang S, Song X, Lin D, Luo R, Shan L, Zhou C, Zhou Z, Zhao Q, Hu C, Hu Y, Guo Q, Chang J, Huang C, Zeng X, Han B, Han X, Jia B, Han Y, Huang Y. China experts consensus on the diagnosis and treatment of advanced stage primary lung cancer (2016 Version). Zhongguo Fei Ai Za Zhi. 2016;19(1):115. https://doi.org/10.3779/j.issn.1009-3419.2016.01.01.

8. Detterbeck FC, Boffa DJ, Kim AW, Tanoue LT. The eighth edition lung Cancer stage classification. Chest. 2017;151(1):193-203. https://doi.org/10. 1016/j.chest.2016.10.010.

9. Evison M, Clive A, Castle L, Powell H, Thomas R, Buttery R, Masani V, Harden S, West D, Woolhouse I. Resectable clinical N2 non-small cell lung Cancer; what is the optimal treatment strategy? An update by the British Thoracic Society lung Cancer specialist advisory group. J Thorac Oncol. 2017;12(9): 1434-41. https://doi.org/10.1016/j.jtho.2017.05.023.
10. Lally BE, Zelterman D, Colasanto JM, Haffty BG, Detterbeck FC, Wilson LD. Postoperative radiotherapy for stage II or III non-small-cell lung cancer using the surveillance, epidemiology, and end results database. J Clin Oncol. 2006; 24(19):2998-3006. https://doi.org/10.1200/JCO.2005.04.6110.

11. Bryan DS, Donington JS. The role of surgery in Management of Locally Advanced non-Small Cell Lung Cancer. Curr Treat Options in Oncol. 2019; 20(4):27. https://doi.org/10.1007/s11864-019-0624-7.

12. Le Pechoux C. Role of postoperative radiotherapy in resected non-small cell lung cancer: a reassessment based on new data. Oncologist. 2011;16(5): 672-81. https://doi.org/10.1634/theoncologist.2010-0150.

13. Thomas M, Rube C, Hoffknecht P, Macha HN, Freitag L, Linder A, Willich N, Hamm M, Sybrecht GW, Ukena D, Deppermann KM, Droge C, Riesenbeck D, Heinecke A, Sauerland C, Junker K, Berdel WE, Semik M, G. German Lung Cancer Cooperative. Effect of preoperative chemoradiation in addition to preoperative chemotherapy: a randomised trial in stage III non-small-cell lung cancer. Lancet Oncol. 2008;9(7):636-48. https://doi.org/10.1016/S14702045(08)70156-6.

14. Shah AA, Berry MF, Tzao C, Gandhi M, Worni M, Pietrobon R, D'Amico TA. Induction chemoradiation is not superior to induction chemotherapy alone in stage IIIA lung cancer. Ann Thorac Surg. 2012;93(6):1807-12. https://doi. org/10.1016/j.athoracsur.2012.03.018.

15. Sonett JR, Suntharalingam M, Edelman MJ, Patel AB, Gamliel Z, Doyle A, Hausner P, Krasna M. Pulmonary resection after curative intent radiotherapy (>59 Gy) and concurrent chemotherapy in non-small-cell lung cancer. Ann Thorac Surg. 2004;78(4):1200-5; discussion 1206. https://doi.org/10.1016/j. athoracsur.2004.04.085

16. Chen D, Wang H, Song $X$, Yue J, Yu J. Preoperative radiation may improve the outcomes of resectable IIIA/N2 non-small-cell lung cancer patients: a propensity score matching-based analysis from surveillance, epidemiology, and end results database. Cancer Med. 2018;7(9):4354-60. https://doi.org/10. 1002/cam4.1701.

17. Pang Z, Yang Y, Ding N, Huang C, Zhang T, Ni Y, Du J, Liu Q. Optimal managements of stage IIIA (N2) non-small cell lung cancer patients: a population-based survival analysis. J Thorac Dis. 2017;9(10):4046-56. https://doi.org/10.21037/jtd.2017.10.47.

18. Wang $S, M a Z$, Yang $X$, Wang $Y, X u Y$, Xia W, Chen R, Qiu M, Jiang $F$, Yin $R, X u L, X u K$. Choice of postoperative radiation for stage IIIA pathologic N2 non-small cell lung cancer: impact of metastatic lymph node number. Radiat Oncol. 2017;12(1):207. https://doi.org/10.1186/ s13014-017-0946-1.

19. Cronin KA, Ries LA, Edwards BK. The surveillance, epidemiology, and end results (SEER) program of the National Cancer Institute. Cancer. 2014; 120(Suppl 23):3755-7. https://doi.org/10.1002/cncr.29049.

20. Park HS, Lloyd S, Decker RH, Wilson LD, Yu JB. Overview of the surveillance, epidemiology, and end results database: evolution, data variables, and quality assurance. Curr Probl Cancer. 2012;36(4):183-90. https://doi.org/10. 1016/j.currproblcancer.2012.03.007.

21. Sanchez-Lorente D, Guzman R, Boada M, Guirao A, Carriel N, Molins L. N2 disease in non-small-cell lung cancer: straight to surgery? Future Oncol. 2018;14(6s):13-6. https://doi.org/10.2217/fon-2017-0387.

22. Suzuki K, Nagai K, Yoshida J, Nishimura M, Takahashi K, Nishiwaki Y. The prognosis of surgically resected N2 non-small cell lung cancer: the importance of clinical N status. J Thorac Cardiovasc Surg. 1999;118(1):14553. https://doi.org/10.1016/S0022-5223(99)70153-4.

23. Regnard JF, Magdeleinat P, Azoulay D, Dartevelle P, Deneuville M, RojasMiranda A, Levasseur P. Results of resection for bronchogenic carcinoma with mediastinal lymph node metastases in selected patients. Eur J Cardiothorac Surg. 1991;5(11):583-6; discussion 587. https://doi.org/10.1016/ 1010-7940(91)90224-8.

24. Massard G, Renaud S, Reeb J, Santelmo N, Olland A, Falcoz PE. N2-IIIA nonsmall cell lung cancer: a plea for surgery! J Thorac Dis. 2016;8(Suppl 11): S849-54. https://doi.org/10.21037/itd.2016.09.34.

25. Veeramachaneni NK, Feins RH, Stephenson BJ, Edwards LJ, Fernandez FG. Management of stage IIIA non-small cell lung cancer by thoracic surgeons in North America. Ann Thorac Surg. 2012;94(3):922-6; discussion 926-8. https://doi.org/10.1016/j.athoracsur.2012.04.087.

26. Caglar HB, Baldini EH, Othus M, Rabin MS, Bueno R, Sugarbaker DJ, Mentzer SJ, Janne PA, Johnson BE, Allen AM. Outcomes of patients with stage III nonsmall cell lung cancer treated with chemotherapy and radiation with and without surgery. Cancer. 2009;115(18):4156-66. https://doi.org/10.1002/ cncr.24492. 
27. Toyooka S, Kiura K, Shien K, Katsui K, Hotta K, Kanazawa S, Date H, Miyoshi S. Induction chemoradiotherapy is superior to induction chemotherapy for the survival of non-small-cell lung cancer patients with pathological mediastinal lymph node metastasis. Interact Cardiovasc Thorac Surg. 2012;15(6):954-60. https://doi.org/10.1093/icvts/ivs412.

28. Koshy M, Fedewa SA, Malik R, Ferguson MK, Vigneswaran WT, Feldman L, Howard A, Abdelhady K, Weichselbaum RR, Virgo KS. Improved survival associated with neoadjuvant chemoradiation in patients with clinical stage IIIA(N2) non-small-cell lung cancer. J Thorac Oncol. 2013;8(7):915-22. https:// doi.org/10.1097/JTO.0b013e31828f68b4.

29. Yamaguchi M, Toyokawa G, Ohba T, Sasaki T, Kometani T, Hamatake M, Hirai F, Taguchi K, Yamanaka T, Seto T, Takenoyama M, Sugio K, Ichinose Y. Preoperative concurrent chemoradiotherapy of S-1/cisplatin for stage III non-small cell lung cancer. Ann Thorac Surg. 2013;96(5):1783-9. https://doi. org/10.1016/j.athoracsur.2013.06.036.

30. Johnstone DW, Byhardt RW, Ettinger D, Scott CB. Phase III study comparing chemotherapy and radiotherapy with preoperative chemotherapy and surgical resection in patients with non-small-cell lung cancer with spread to mediastinal lymph nodes (N2); final report of RTOG 89-01. Radiation therapy oncology group. Int J Radiat Oncol Biol Phys. 2002;54(2):365-9.

31. Katakami N, Tada H, Mitsudomi T, Kudoh S, Senba H, Matsui K, Saka H, Kurata T, Nishimura Y, Fukuoka M. A phase 3 study of induction treatment with concurrent chemoradiotherapy versus chemotherapy before surgery in patients with pathologically confirmed N2 stage IIIA nonsmall cell lung cancer (WJTOG9903). Cancer. 2012;118(24):6126-35. https://doi.org/10.1002/ cncr.26689.

32. Pless M, Stupp R, Ris HB, Stahel RA, Weder W, Thierstein S, Gerard MA, Xyrafas A, Fruh M, Cathomas R, Zippelius A, Roth A, Bijelovic M, Ochsenbein A, Meier UR, Mamot C, Rauch D, Gautschi O, Betticher DC, Mirimanoff RO, Peters S, S.L.C.P. Group. Induction chemoradiation in stage IIIA/N2 nonsmall-cell lung cancer: a phase 3 randomised trial. Lancet. 2015;386(9998): 1049-56. https://doi.org/10.1016/S0140-6736(15)60294-X.

33. Yang CF, Gulack BC, Gu L, Speicher PJ, Wang X, Harpole DH, Onaitis MW, D'Amico TA, Berry MF, Hartwig MG. Adding radiation to induction chemotherapy does not improve survival of patients with operable clinical N2 non-small cell lung cancer. J Thorac Cardiovasc Surg. 2015;150(6):148492; discussion 1492-3. https://doi.org/10.1016/j.jtcvs.2015.06.062.

34. Girard N, Mornex F, Douillard JY, Bossard N, Quoix E, Beckendorf V, Grunenwald D, Amour E, Milleron B. Is neoadjuvant chemoradiotherapy a feasible strategy for stage IIIA-N2 non-small cell lung cancer? Mature results of the randomized IFCT-0101 phase II trial. Lung Cancer. 2010;69(1):86-93. https://doi.org/10.1016/j.lungcan.2009.10.003

35. Mirimanoff RO. Neoadjuvant chemoradiotherapy followed by surgery for stage IIla and IIlb non-small-cell lung cancer (NSCLC): is it still justified? Chin Clin Oncol. 2015;4(4):49. https://doi.org/10.3978/j.issn.2304-3865.2015.12.05.

36. Rigotti NA. Strategies to help a smoker who is struggling to quit. JAMA. 2012:308(15):1573-80. https://doi.org/10.1001/jama.2012.13043.

37. Driessen EJM, Janssen-Heijnen MLG, Maas HA, Dingemans AC, van Loon JGM. Study protocol of the NVALT25-ELDAPT trial: selecting the optimal treatment for older patients with stage III non-small-cell lung Cancer. Clin Lung Cancer. 2018;19(6):e849-52. https://doi.org/10.1016/j.cllc.2018.07.003.

38. Schulkes KJ, Hamaker ME, van den Bos F, van Elden LJ. Relevance of a geriatric assessment for elderly patients with lung Cancer-a systematic review. Clin Lung Cancer. 2016;17(5):341-9 e3. https://doi.org/10.1016/j.cllc. 2016.05.007.

39. Mellemgaard A, Luchtenborg M, lachina M, Jakobsen E, Green A, Krasnik M, Moller $\mathrm{H}$. Role of comorbidity on survival after radiotherapy and chemotherapy for nonsurgically treated lung cancer. J Thorac Oncol. 2015; 10(2):272-9. https://doi.org/10.1097/JTO.0000000000000416.

40. Semrau S, Zettl H, Hildebrandt G, Klautke G, Fietkau R. Older patients with inoperable non-small cell lung cancer: long-term survival after concurrent chemoradiotherapy. Strahlenther Onkol. 2014;190(12):1125-32. https://doi. org/10.1007/s00066-014-0710-5.

41. Kelly RJ, Rajan A, Force J, Lopez-Chavez A, Keen C, Cao L, Yu Y, Choyke P, Turkbey B, Raffeld M, Xi L, Steinberg SM, Wright JJ, Kummar S, Gutierrez M, Giaccone $\mathrm{G}$. Evaluation of KRAS mutations, angiogenic biomarkers, and DCE-MRI in patients with advanced non-small-cell lung cancer receiving sorafenib. Clin Cancer Res. 2011;17(5):1190-9. https://doi.org/10.1158/10780432.CCR-10-2331.

42. Eberhardt WE, Mitchell A, Crowley J, Kondo H, Kim YT, Turrisi A 3rd, Goldstraw P, Rami-Porta R, S. International Association for Study of Lung
Cancer, A.B.M. Prognostic Factors Committee, and I. Participating. The IASLC lung Cancer staging project: proposals for the revision of the $\mathrm{M}$ descriptors in the forthcoming eighth edition of the TNM classification of lung Cancer. J Thorac Oncol. 2015;10(11):1515-22. https://doi.org/10.1097/JTO. 0000000000000673

43. Groome PA, Bolejack V, Crowley JJ, Kennedy C, Krasnik M, Sobin LH, Goldstraw P, Committee IIS, Cancer R, Biostatistics, C. Observers to the, and I. Participating. The IASLC lung Cancer staging project: validation of the proposals for revision of the T, N, and M descriptors and consequent stage groupings in the forthcoming (seventh) edition of the TNM classification of malignant tumours. J Thorac Oncol. 2007;2(8):694-705. https://doi.org/10. 1097/JTO.0b013e31812d05d5.

44. Ball D, Mitchell A, Giroux D, Rami-Porta R, Committee IS, Participating I. Effect of tumor size on prognosis in patients treated with radical radiotherapy or chemoradiotherapy for non-small cell lung cancer. An analysis of the staging project database of the International Association for the Study of Lung Cancer. J Thorac Oncol. 2013;8(3):315-21. https://doi.org/ 10.1097/JTO.0b013e31827dc74d.

\section{Publisher's Note}

Springer Nature remains neutral with regard to jurisdictional claims in published maps and institutional affiliations.
Ready to submit your research? Choose BMC and benefit from:

- fast, convenient online submission

- thorough peer review by experienced researchers in your field

- rapid publication on acceptance

- support for research data, including large and complex data types

- gold Open Access which fosters wider collaboration and increased citations

- maximum visibility for your research: over $100 \mathrm{M}$ website views per year

At BMC, research is always in progress.

Learn more biomedcentral.com/submissions 